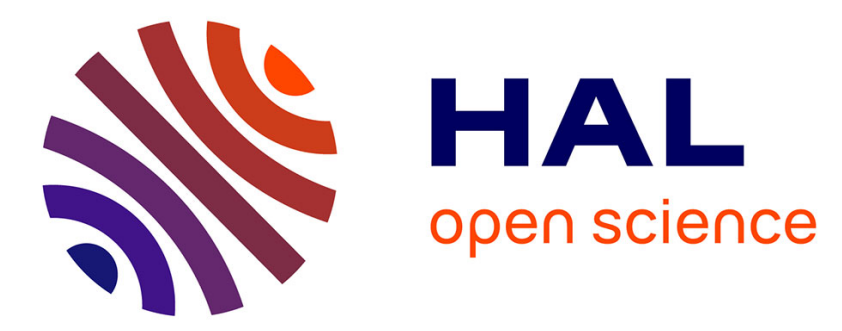

\title{
Un spectromètre à haute résolution pour la détermination des sections efficaces différentielles en angle et énergie de choc d'ions lents $(0,5$ a $3 \mathrm{keV})$ sur des gaz
}

\author{
A. Pernot, M. Abognoli, M. Barat, J. Baudon, A. Septier
}

\section{To cite this version:}

A. Pernot, M. Abognoli, M. Barat, J. Baudon, A. Septier. Un spectromètre à haute résolution pour la détermination des sections efficaces différentielles en angle et énergie de choc d'ions lents (0,5 a $3 \mathrm{keV}$ ) sur des gaz. Revue de Physique Appliquée, 1967, 2 (3), pp.203-212. 10.1051/rphysap:0196700203020300 . jpa-00242792

\section{HAL Id: jpa-00242792 https://hal.science/jpa-00242792}

Submitted on 1 Jan 1967

HAL is a multi-disciplinary open access archive for the deposit and dissemination of scientific research documents, whether they are published or not. The documents may come from teaching and research institutions in France or abroad, or from public or private research centers.
L'archive ouverte pluridisciplinaire HAL, est destinée au dépôt et à la diffusion de documents scientifiques de niveau recherche, publiés ou non, émanant des établissements d'enseignement et de recherche français ou étrangers, des laboratoires publics ou privés. 


\title{
UN SPEGTROMÈTRE A HAUTE RÉSOLUTION POUR LA DÉTERMINATION DES SEGTIONS EFFIGAGES DIFFÉRENTIELLES EN ANGLE ET ÉNERGIE DE GHOG D'IONS LENTS (0,5 A 3 keV) SUR DES GAZ
}

\author{
Par A. PERNOT (1), M. ABIGNOLI (2), M. BARAT, J. BAUDON et A. SEPTIER, \\ Institut d'Électronique Fondamentale, Laboratoire associé au G.N.R.S., \\ Faculté des Sciences d'Orsay, Bât. 220, 91-Orsay.
}

\begin{abstract}
Résumé. - Grâce au spectromètre décrit ici, les ions d'énergie comprise entre 500 et $3000 \mathrm{eV}$, diffusés par collision avec des atomes ou des molécules, peuvent être analysés en angle, en énergie et en masse. Ainsi on peut déterminer les sections efficaces différentielles soit pour un processus élastique ou inélastique, grâce à l'analyse en énergie, soit pour un processus de réarrangement, en utilisant le sélecteur de masse.
\end{abstract}

Abstract. - A spectrometer is described, in which ions (energy range 500-3 $000 \mathrm{eV}$ ) scattered by atoms and molecules are analyzed versus angle, energy and mass. In this way differential cross sections (elastic or inelastic by energy analysis, rearrangement by a supplementary mass analysis) can be determined.

Introduction. - L'étude expérimentale précise des mécanismes de collision nécessite la détermination des sections efficaces différentielles angulaires pour un processus déterminé, élastique ou non. En effet, en raisonnant classiquement, ce qui est justifié pour des particules lourdes d'énergie au moins égale à quelques dizaines d'eV, à un couple de valeurs $E$ (énergie), $\theta$ (angle de diffusion) correspond une valeur déterminée de la distance $r_{0}$ de plus petite approche : la collision considérée est donc parfaitement déterminée.

Il est possible en particulier de déduire des mesures différentielles les courbes de potentiel d'interaction ion-atome ainsi que les mécanismes d'échange ou d'excitation, et de vérifier expérimentalement les calculs théoriques, du moins pour les systèmes les plus simples (hydrogène et hélium).

La détermination des sections différentielles, souvent effectuée dans le cas de la diffusion électronique, n'a été entreprise qu'assez récemment pour les collisions ion-atome. Elle a déjà permis de déterminer certains potentiels d'interaction aux faibles distances internucléaires [1]. D'autres mesures [2] effectuées à basse énergie avec une résolution angulaire élevée, sur la collision élastique $\mathrm{He}^{+}-\mathrm{He}$, ont mis en évidence des « structures résonnantes ", résultant de l'interférence des ondes diffusées par les potentiels « pair » et « impair » de l'ion moléculaire $\mathrm{He}_{2}^{+}$, ainsi que des oscillations secondaires provenant de l'échange des noyaux.

(1) La réalisation et la mise au point de ce spectromètre ont été effectuées dans le cadre de la thèse de DocteurIngénieur de $M$. A. Pernot.

(2) Faculté des Sciences de Paris.
L'appareil décrit dans le présent article permet de déterminer les sections efficaces différentielles (élastiques et inélastiques) dans le domaine d'énergie $500 \mathrm{eV}-3000 \mathrm{eV}$. Reprenant les mesures d'Aberth et Lorents à des énergies plus élevées, nous avons pu mettre en évidence les mêmes structures résonnantes.

D'autre part, l'analyse en masse des particules diffusées permet une étude angulaire de la diffusion avec réarrangement. Nous pensons, en particulier, reprendre à plus basse énergie les expériences de McClure [3] sur la dissociation de $\mathrm{H}_{2}^{+}$.

1. Description de l'appareil ( fig. 1). - 1.1. ProducTION DU FAISGEAU D'IONS INGIDENT. - Les ions projectiles sont produits par une source du type PIG Reflex à cathode chaude, fonctionnant à basse pression (quelque $10^{-4}$ torr), dérivée d'une réalisation de Garlson et Magnuson [4]. L'utilisation d'une tension d'arc peu élevée et stabilisée (90 V dans l'hélium, $40 \mathrm{~V}$ dans l'argon) assure au faisceau extrait une faible dispersion en énergie $(2 \pm 1 \mathrm{eV})$. La cathode chaude est une spirale plane en fil de tantale (diamètre $0,8 \mathrm{~mm}$ ) chauffée en courant continu (15 à $20 \mathrm{~A})$. Les électrons émis oscillent entre la cathode chaude et la cathode froide, qui est percée du trou d'extraction (diamètre $3 \mathrm{~mm}$ ). Un champ magnétique axial important (0 à $2000 \mathrm{G})$ a pour rôle d'accroître considérablement la longueur des trajectoires électroniques entre cathodes et anode. L'ensemble de la source est porté à la tension correspondant à l'énergie de collision choisie $E$. L'électrode d'extraction, de forme conique, se trouve au potentiel de $-2500 \mathrm{~V}$ par rapport à la source. Pour une tension d'extraction aussi faible, l'ouverture angulaire du faisceau extrait est importante : un diaphragme en réduit la valeur à $\pm 6^{\circ}$. 


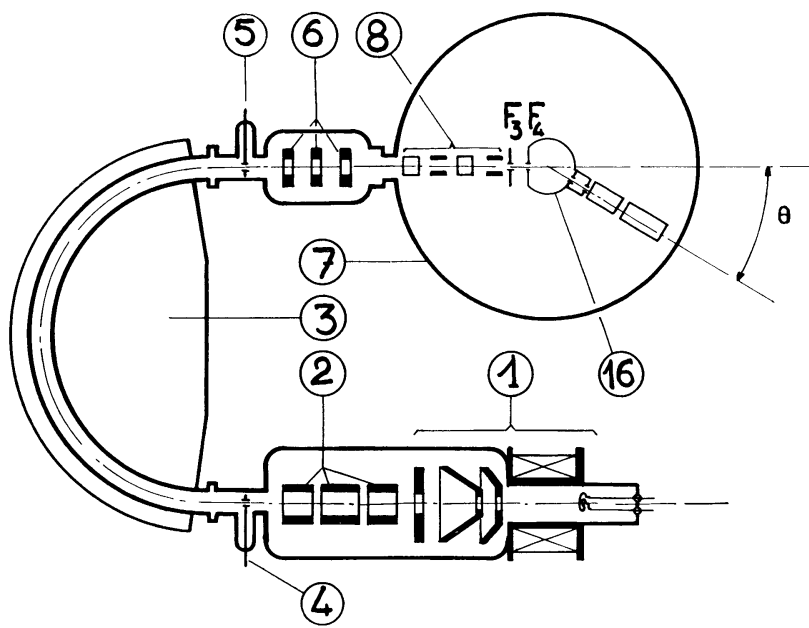

FIG. 1. - Schéma général. - Vue de dessus : 1. Source d'ions. - 2. Lentille électrostatique de focalisation. - 3. Monochromateur $180^{\circ}$. - 4. Fente d'entrée du monochromateur $\mathrm{F}_{1}$. -5 . Fente de sortie du monochromateur $\mathrm{F}_{2}$. -6 . Lentille électrostatique de focalisation. - 7. Enceinte de l'analyseur. - 8. Système de déflecteurs électrostatiques. - 16. Chambre de collisions.

Le faisceau, qui peut être éventuellement ralenti après extraction, est focalisé sur la fente d'entrée $F_{1}$ du monochromateur, par une lentille électrostatique formée de trois électrodes cylindriques.

Le courant extrait est de quelques centaines de $\mu \mathrm{A}$, mais le diaphragme ramène l'intensité du faisceau provenant de la fente $F_{1}$ aux environs de $10 \mu \mathrm{A}$. La distribution radiale de la densité de courant dans une section du faisceau présente un maximum aigu sur l'axe qui dépend de façon critique de la valeur du champ magnétique dans la source. Comme seule la partie centrale du faisceau est utilisée, il est nécessaire de bien stabiliser le champ magnétique, pour obtenir un courant ionique aussi stable que possible. Cette source permet d'obtenir des faisceaux d'ions hydrogène, ou de différents gaz rares.

1.2. Le monochromateur. - 1.2.1. Double focalisation par champ magnétique non uniforme. - Un secteur magnétique à $180^{\circ}$, placé après la source, a le double rôle de sélectionner des particules de rapport $\mathrm{q} / \mathrm{m}$ déterminé et de réduire éventuellement la dispersion en énergie propre du faisceau. Un champ magnétique non uniforme $B$ assure une double focalisation, à la fois dans le plan horizontal et dans le plan vertical.

En coordonnées cylindriques $(r, \theta, z)$, les composantes de B sont :

$$
B_{z}=B_{0}\left(1-n \frac{r-r_{0}}{r_{0}}\right) \quad \text { et } \quad B_{r}=-B_{0} n \frac{z}{r_{0}}
$$

(voir fig. 2)

où $r_{0}$ est le rayon de la trajectoire circulaire moyenne, $n$ est appelé l'indice du champ.

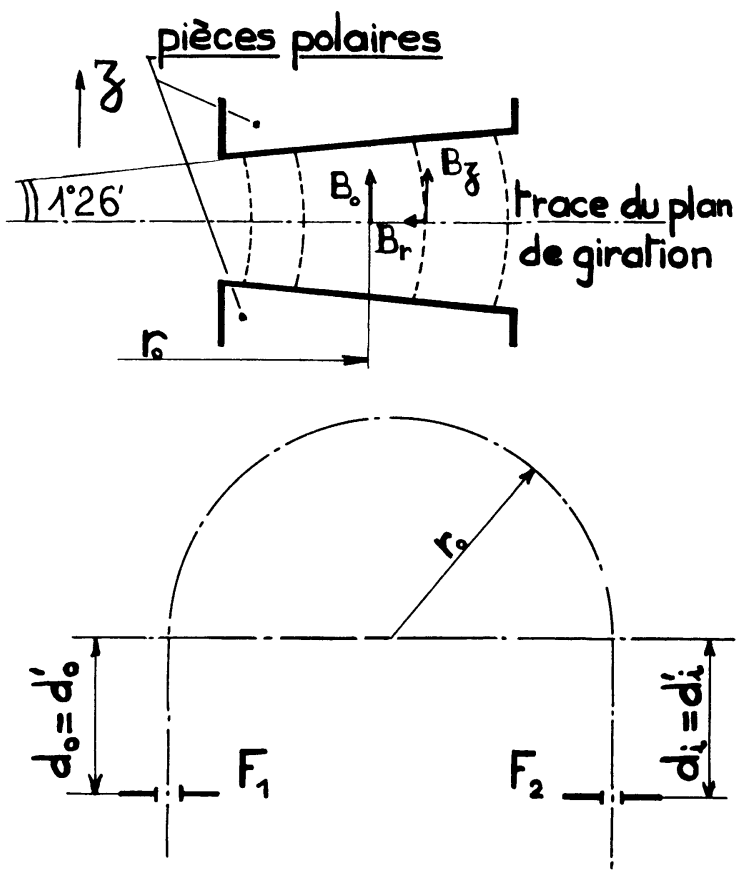

FIG. 2. - Optique du monochromateur.

Considérons une trajectoire incidente caractérisée dans le plan de la face d'entrée du secteur par

$$
\begin{aligned}
x_{0} & =\left(r-r_{0}\right)_{\text {initial }}, & & z_{0} \\
x_{0}^{\prime} & =\left[\frac{\mathrm{d}}{\mathrm{d} s}\left(r-r_{0}\right)\right]_{\text {initial }}, & & z_{0}^{\prime}=\left(\frac{\mathrm{d} z}{\mathrm{~d} s}\right)_{\text {initial }}
\end{aligned}
$$

$s$ étant l'abscisse curviligne. Il lui correspond une trajectoire émergente caractérisée de même par $x_{\mathrm{i}}, x_{\mathrm{i}}^{\prime}$, $z_{i}, z_{\mathrm{i}}^{\prime}$. Le passage de l'espace objet (o) à l'espace image (i) s'écrit à l'aide des matrices de transfert :

$$
\left\{\begin{array}{l}
\left(\begin{array}{l}
x_{\mathrm{i}} \\
x_{\mathrm{i}}^{\prime}
\end{array}\right)=\left[\begin{array}{cc}
\cos \frac{\sqrt{1-n}}{r_{0}} L & \frac{r_{0}}{\sqrt{1-n}} \sin \frac{\sqrt{1-n}}{r_{0}} L \\
-\frac{\sqrt{1-n}}{r_{0}} \sin \frac{\sqrt{1-n}}{r_{0}} L & \cos \frac{\sqrt{1-n}}{r_{0}} L
\end{array}\right]\left(\begin{array}{l}
x_{0} \\
x_{0}^{\prime}
\end{array}\right) \\
\left(\begin{array}{c}
z_{\mathrm{i}} \\
z_{\mathrm{i}}^{\prime}
\end{array}\right)=\left[\begin{array}{cc}
\cos \frac{\sqrt{n}}{r_{0}} L & \frac{r_{0}}{\sqrt{n}} \sin \frac{\sqrt{n}}{r_{0}} L \\
-\frac{\sqrt{n}}{r_{0}} \sin \frac{\sqrt{n}}{r_{0}} L & \cos \frac{\sqrt{n}}{r_{0}} L
\end{array}\right]\left(\begin{array}{l}
z_{0} \\
z_{0}^{\prime}
\end{array}\right)
\end{array}\right.
$$


où $L$ représente la longueur de la trajectoire moyenne entre les faces d'entrée et de sortie.

Considérons les points antinodaux $\mathrm{A}_{0} \mathrm{~A}_{\mathrm{i}}$ pour le plan horizontal (rox), $\mathrm{A}_{0}^{\prime} \mathrm{A}_{\mathrm{i}}^{\prime}$ pour le plan vertical (roz). Ces points sont les foyers du secteur à $90^{\circ}$. Soient $d_{0}$ et $d_{0}^{\prime}$ les distances de $A_{0}$ et $A_{0}^{\prime}$ à la face d'entrée, $d_{\mathrm{i}}$ et $d_{\mathrm{i}}^{\prime}$ les distances de la face de sortie à $A_{\mathrm{i}}$ et $A_{\mathrm{i}}^{\prime}$. On obtient :

$$
\begin{gathered}
d_{0}=d_{\mathrm{i}}=\frac{r_{0}}{\sqrt{1-n} \operatorname{tg} \frac{\pi}{2} \sqrt{1-n}} \\
d_{0}^{\prime}=d_{\mathrm{i}}^{\prime}=\frac{r_{0}}{\sqrt{n} \operatorname{tg} \frac{\pi}{2} \sqrt{n}} .
\end{gathered}
$$

La double focalisation impose $d_{0}=d_{0}^{\prime}$ et $d_{\mathrm{i}}=d_{\mathrm{i}}^{\prime}$, donc $\sqrt{n}=\sqrt{1-n}$, soit $n=\frac{1}{2}$.

Un tel champ magnétique est obtenu théoriquement en utilisant des pièces polaires de section hyperbolique. Mais lorsque le faisceau n'a pas une extension radiale trop grande, on peut obtenir ce champ avec une très bonne approximation dans la zone utile, en remplaçant les hyperboles par des droites : les pôles sont alors des sections de cônes.

On calcule simplement l'angle $u$ que doivent faire les faces de l'entrefer avec le plan horizontal, par la relation $\operatorname{tg} u=n \cdot \frac{e}{r_{0}}$, où $e$ est la demi-largeur de l'entrefer en $r=r_{0}(=30 \mathrm{~cm})$. On trouve ici $u=1^{\circ} 26^{\prime}$. Cette valeur théorique ne peut être réalisée en pratique que de façon approchée ; d'autre part, les caractéristiques magnétiques du fer peuvent varier légèrement d'un bout à l'autre des pôles : il est donc très difficile de réaliser un « indice » $n$ rigoureusement égal à $\frac{1}{2}$ tout au long de l'aimant. On déduit alors des formules précédentes, avec $r_{0}=30 \mathrm{~cm}$, $d_{0}=d_{0}^{\prime}=d_{\mathrm{i}}=d_{\mathrm{i}}^{\prime} \simeq 21 \mathrm{~cm}$, ce qui fixe la position des fentes d'entrée $F_{1}$ et de sortie $F_{2}$ du monochromateur.

La réalisation mécanique du monochromateur a été inspirée de celle de Snyder et al. [6].

La valeur maximum de champ magnétique $(1,8 \mathrm{kG})$ permet la sélection d'ions de $3000 \mathrm{eV}$ jusqu'à la masse $\left(\mathrm{Kr}^{+}\right)$.

1.2.2. Optique entre monochromateur et chambre de collision. - Le faisceau issu de $\mathrm{F}_{2}$ est rendu parallèle à l'axe par une deuxième lentille électrostatique et pénètre dans la chambre de collisions par les fentes collimatrices $\mathrm{F}_{3}$ et $\mathrm{F}_{4}$.

Un double système de plaques déflectrices parallèles ( fig. 3), agissant respectivement dans les plans vertical et horizontal, permet d'ajuster avec précision la position et la direction du faisceau dans la chambre de collision, par effet de « lame à faces parallèles » $\left(\mathrm{P}_{1}, \mathrm{P}_{2}\right)$ ou par effet de « prisme » $\left(\mathrm{P}_{3}, \mathrm{P}_{4}\right)$.

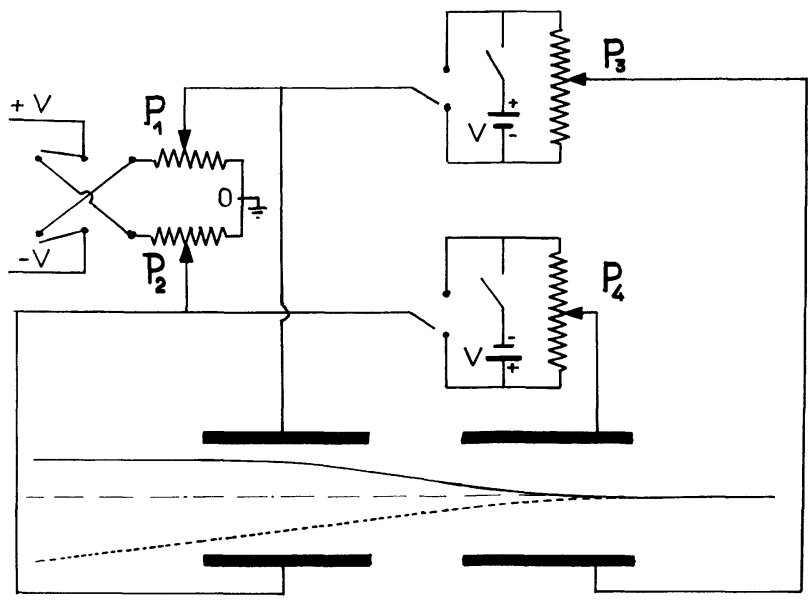

FIG. 3. - Schéma d'alimentation des déflecteurs électrostatiques.

1.2.3. Vide. - La pression réalisée dans l'enceinte de la source et du monochromateur est de $4 \times 10^{-7}$ torr (en vide limite), et de 5 à $6 \times 10^{-6}$ torr lorsque la source est alimentée en gaz à une pression de $10^{-4}$ torr ; le groupe de pompage comprend deux pompes à diffusion d'huile, l'une d'un débit de $600 \mathrm{1} / \mathrm{s}$ pour la source, l'autre de $150 \mathrm{l} / \mathrm{s}$ pour le monochromateur. Ces pompes sont munies de baffles refroidis à l'azote liquide. Les joints utilisés sont de qualité « viton ».

1.3. Analyseur Proprement dit (fig. 4). - L'ensemble analyseur se trouve dans une enceinte en acier inoxydable dans laquelle est réalisée une pression de $6 \times 10^{-8}$ torr en vide limite, et de $10^{-6}$ torr lorsque la chambre de collision est alimentée en gaz cible à une pression de $10^{-3}$ Torr.

Les différents éléments constituant l'analyseur sont en acier inoxydable et, pour éviter les instabilités de faisceau, toutes les électrodes sont plaquées or et les fentes sont recouvertes d'aquadag. Toutes les parties isolantes ont été réalisées en quartz, et sont cachées à la « vue »du faisceau.

Les ions ayant subi des collisions avec le gaz cible, ou les ions secondaires formés lors d'un choc, sont analysés d'abord en énergie, dans toutes les directions d'émergence, à l'aide d'un secteur électrostatique, puis en masses, à l'aide d'un petit électroaimant placé à la suite du secteur électrostatique. Ils sont ensuite comptés par le détecteur.

Les éléments de l'analyseur fixés sur une potence verticale sont solidaires d'un plateau tournant horizontal reposant sur un chemin de roulement à billes et entraîné magnétiquement de l'extérieur de l'enceinte par trois électroaimants faisant face à des masses d'acier doux. Le plateau est gradué en degrés et un hublot permet de lire à $6^{\prime}$ d'arc près l'angle $\theta$ que fait le plan de symétrie vertical du système d'analyse avec la direction du faisceau incident. 


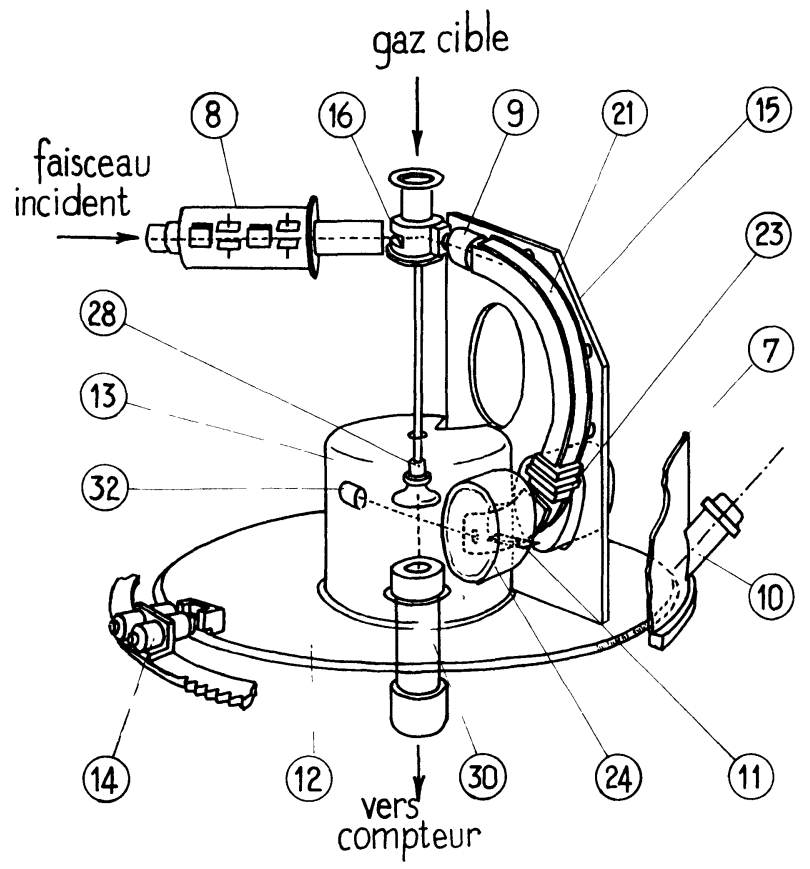

Frg. 4. - Schéma général de l'analyseur : 7. Enceinte de l'analyseur. - 8. Déflecteurs électrostatiques. 9. Système $L_{1}$. - 10. Hublot de visée. - 11. Collecteur. - 12. Plateau tournant. - 13. Blindage. - 14. Système d'entraînement. - 15. Potence verticale. 16. Chambre de collisions.

1.3.1. Chambre de collisions. - La chambre de collisions ( fig. 5) est formée de deux cylindres coaxiaux dont l'axe coïncide avec celui du plateau tournant, ajustés à frottement doux; les surfaces en contact sont rectifiées et la surface de la partie interne est recouverte de chrome dur pour éviter tout risque de grippage.

Dans ces conditions, le rapport des pressions entre

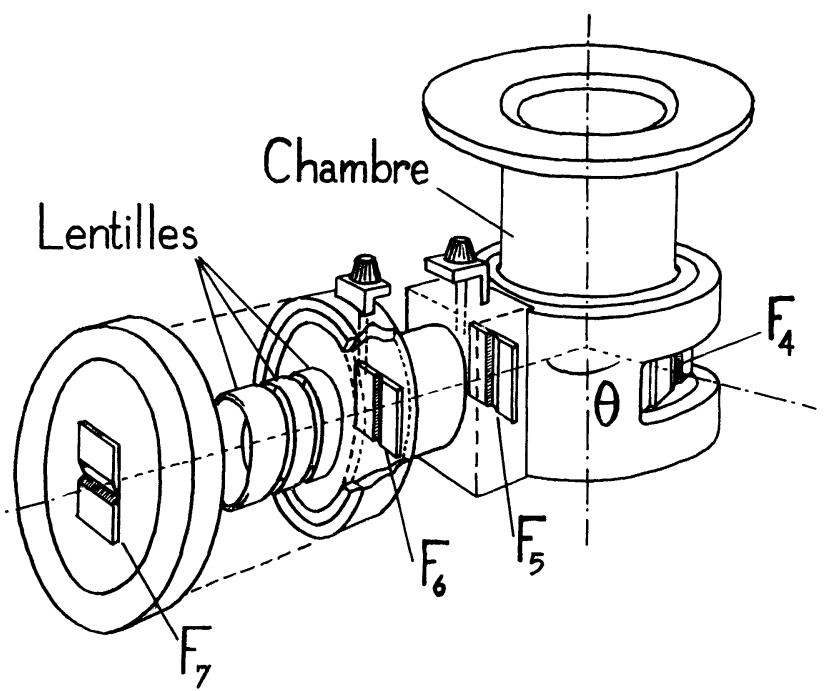

FrG. 5. - Chambre de collisions. Système optique. la chambre de collision etl'analyseur est supérieur à 100 . Le cylindre extérieur porte deux fentes identiques $\mathrm{F}_{5}$ et $F_{6}$ définissant la direction d'émergence $\theta$, distantes de $40 \mathrm{~mm}$ et de largeur ajustable par vis micrométrique.

Une petite cage de Faraday escamotable permet de mesurer le courant d'ions pénétrant dans la chambre $\left(5 \times 10^{-8} \mathrm{~A}\right.$ environ, pour une énergie incidente de $2 \mathrm{keV})$.

1.3.2. Système optique $\mathrm{L}_{1}$ (Lentilles, fig. 5). - Ge système optique a pour rôle de focaliser sur la fente d'entrée $F_{7}$ du secteur électrostatique les ions sortant de la chambre de collision par les fentes $\mathrm{F}_{5}$ et $\mathrm{F}_{6}$, et de les accélérer (ou de les ralentir) jusqu'à une énergie déterminée. Les propriétés optiques de $\mathrm{L}_{1}$ ont été déterminées par le calcul de quelques trajectoires paraxiales, à partir de la carte de potentiel établie à la cuve rhéographique. Cette étude a permis de conclure que le fait d'accélérer ou de ralentir le faisceau ionique par une tension $V_{1}$ (positive ou négative), à la sortie immédiate de la chambre et dans un espace très restreint, substitue à l'objet une image pratiquement confondue avec lui. Les électrodes de $\mathrm{L}_{1}$ étant alimentées à partir de la tension $V_{1}$, ses propriétés restent inchangées lorsque $V_{1}$ varie, et l'image définitive garde une position fixe.

Le réglage de $L_{1}$ s'effectue en utilisant le faisceau incident parallèle issu de $F_{6}$, pour $\theta=0$. Comme $F_{6}$ est une fente verticale et $F_{7}$ une fente horizontale, $\mathrm{F}_{7}$ doit se trouver au foyer image du système $\mathrm{L}_{1}$. Pour $\theta \neq 0$, le système reçoit tous les ions diffusés passant par $F_{5}$ et $F_{6}$, mais seuls peuvent traverser $F_{7}$ les ions ayant subi une déviation verticale inférieure à $\beta=\frac{w_{7}}{2 f_{1}}$ où $w_{7}(=0,3 \mathrm{~mm})$ est la largeur de $F_{7}$ et $\mathrm{f}_{1}(=60 \mathrm{~mm})$ la distance focale de $\mathrm{L}_{1}$; on obtient $\beta=2,5 \times 10^{-3} \mathrm{rd}$.

La définition angulaire $\delta \theta$ donnée par $\mathrm{F}_{5} \mathrm{~F}_{6}$ est liée à la largeur des fentes et à leur écartement et non à leur hauteur. Toutefois, celle-ci intervient dans la résolution finale de l'analyseur, puisque l'ouverture angulaire dans le plan vertical du faisceau traversant $\mathrm{F}_{7}$ est proportionnelle à la hauteur $h$ de $\mathrm{F}_{5}$ et $\mathrm{F}_{6}$ et intervient dans le pouvoir de résolution de l'analyseur électrostatique. Pour cette raison, la hauteur des fentes a été limitée à 2,5 mm. L'emploi de telles fentes, au lieu de diaphragmes circulaires de diamètre égal à leur largeur, assurant la même définition angulaire, est particulièrement avantageux, puisque le volume de collision d'où sont issus les ions diffusés recueillis dans l'analyseur se trouve multiplié par 10 environ, ce qui représente un gain de 10 sur le courant analysé.

1.3.3. Analyseur électrostatique. - C'est un secteur électrostatique cylindrique d'angle $127^{\circ}$. Pour cette valeur particulière de l'angle de déviation, l'image de la fente d'entrée $F_{7}\left(0,3 \times 5 \mathrm{~mm}^{2}\right)$ se trouve sur la fente de sortie $\mathrm{F}_{8}\left(0,3 \times 5 \mathrm{~mm}^{2}\right)$. Les deux électrodes déflectrices sont des portions de cylindre de rayons $R_{1}=144 \mathrm{~mm}$ et $R_{2}=136 \mathrm{~mm}$; elles sont portées 
respectivement aux tensions $+V_{\mathrm{a}} / 2$ et $-V_{\mathrm{a}} / 2$ par rapport à la tension $V_{1}$. Deux plaques verticales ( fig. 6) parallèles ferment latéralement le secteur cylindrique;

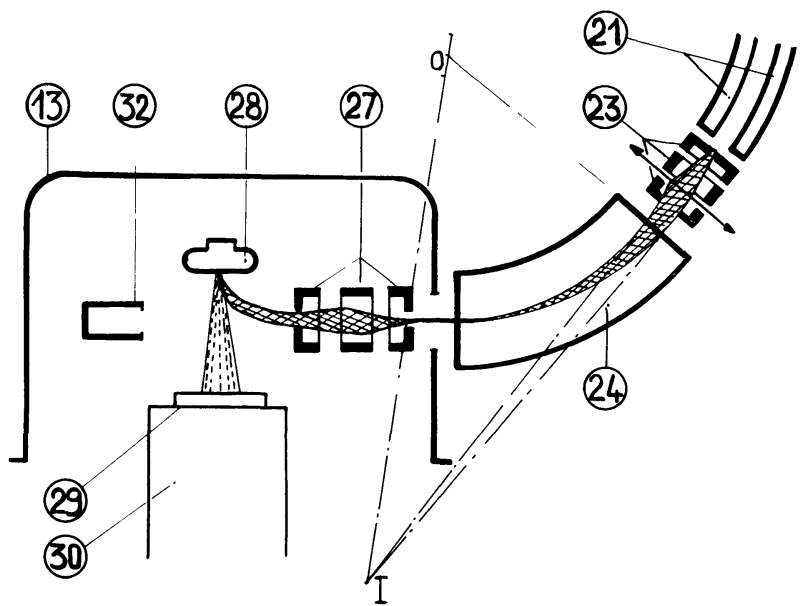

Fig. 6. - Schéma de l'analyseur de masses et du détecteur : 13. Blindage. - 21. Analyseur électrostatique. - 23. Lentille électrostatique de focalisation. 24. Analyseur de masses. - 27. Électrodes de postaccélération. - 28. Cible à émission secondaire. 29. Scintillateur. - 30. Photomultiplicateur. 32. Cage de Faraday finale.

portées à une même tension positive, elles focalisent le faisceau perpendiculairement au plan de la trajectoire.

Le secteur électrostatique est destiné à l'analyse en énergie des ions diffusés, quelles que soient leurs masses respectives. Si l'on désigne par $E$ l'énergie des ions monochargés sortant par $F_{8}$, la différence de potentiel $V_{\mathrm{a}}$ entre les électrodes est reliée à $E$ par l'expression :

$$
V_{\mathrm{a}}(\text { volts })=k E_{(\mathrm{eV})} \quad \text { où } \quad k=2 \log \frac{R_{1}}{R_{2}} \simeq 0,114
$$

(valeur expérimentale : 0,112 ).

La résolution en énergie d'un tel dispositif est donnée par la relation [7] :

$$
\frac{\Delta E}{E}=\frac{4}{3} \alpha^{2}+\frac{1}{2}\left(\frac{h_{7}+h_{8}}{\pi R_{0}}\right)^{2}+\frac{w_{7}+w_{8}}{R}
$$

où $\alpha$ est l'ouverture angulaire du faisceau à l'entrée dans le plan de symétrie de l'analyseur, $h_{7}$ et $h_{8}(5 \mathrm{~mm})$ les longueurs des fentes $F_{7}$ et $F_{8}, w_{7} w_{8}$ leurs largeurs $(0,3 \mathrm{~mm}), R_{0}$ le rayon moyen de la trajectoire $\left(R_{0}=\right.$ $140 \mathrm{~mm}$ ). On trouve pour un angle $\alpha$ de $2,5 \times 10^{-2} \mathrm{rd}$ :

$$
\frac{\Delta E}{E} \simeq 5,3 \times 10^{-3} \text { (à la base du pic). }
$$

1.3.4. Focalisation et analyse magnétique (voir fig. 6). - Les analyseurs électrostatique et magnétique étant très rapprochés, la fente $F_{8}$ est située trop près de l'entrée du secteur magnétique pour que celui-ci en donne une image réelle : il est nécessaire d'assurer une focalisation supplémentaire pour concentrer les ions sur la fente d'entrée $F_{9}$ du détecteur final.

La construction de Cartan relative au prisme magnétique indique que l'objet - qui est virtuel - devra se trouver en I (voir fig. 6). La lentille devra donc donner de $F_{8}$ une image en ce point. On détermine alors la distance focale $f=2 \mathrm{~cm}$. Dans ces conditions, le grandissement linéaire est grand $(\simeq 10)$. La fente $F_{9}$ doit donc être largement ouverte $(1 \mathrm{~mm})$. Il n'y a pas en pratique de perte de résolution car les ions sont déjà, à cet endroit, analysés en énergie, donc monocinétiques, et le secteur magnétique n'a qu'un rôle de sélecteur de masses. Les bobines de l'aimant sont placées, à la pression atmosphérique, dans deux boîtiers étanches, ce qui évite tout dégazage intempestif des enroulements. L'entrefer est de $1 \mathrm{~cm}$, et l'angle de déviation $53^{\circ}$ : le faisceau émergent est de nouveau horizontal. L'ensemble de l'électroaimant est isolé et porté à la tension $V_{1}$.

Le secteur magnétique peut analyser des ions d'énergie $1500 \mathrm{eV}$ jusqu'à la masse 40 (ions $\mathrm{Ar}^{+}$). Lorsque l'électroaimant n'est pas excité, les ions issus de $F_{8}$ sont recueillis par un collecteur auxiliaire, ce qui permet un réglage préliminaire de l'analyseur électrostatique, en utilisant le faisceau direct venant de la source.

1.3.5. Détecteur. - Le courant final diffusé aux grands angles $\theta$ étant extrêmement faible, il est nécessaire d'utiliser un détecteur de très grande sensibilité, capable de compter quelques ions par seconde (voir Appendice I). Nous avons choisi un détecteur mis au point simultanément par Daly et Afrosimov [8].

Les ions issus de $F_{9}$ sont attirés et accélérés par une électrode plane, en acier inoxydable (voir fig. 6), d'axe vertical perpendiculaire à la direction primitive du faisceau, et portée à une tension négative de $25 \mathrm{kV}$. Les ions issus de $\mathrm{F}_{9}$ sont déviés vers le centre de l'électrode et provoquent l'émission d'électrons secondaires qui se trouvent alors accélérés vers la surface métallisée d'un cristal scintillateur à une énergie de $25 \mathrm{keV}$. Lors d'une étude sur cette technique de détection [9], on a montré que divers métaux (aluminium et argent en particulier), déposés en couche mince sur l'électrode d'acier inox, améliorent son coefficient d'émission secondaire d'un facteur 2 à 3 . Le scintillateur plastique (Radiotechnique-SPF) est accolé à un hublot en verre optique qui assure l'étanchéité tout en laissant passer la lumière en provenance du scintillateur. Le photomultiplicateur est appliqué au hublot : le contact optique est assuré par une couche de graisse à vide. Ce photomultiplicateur à très faible bruit (EMI 9514S) permet alors de compter les électrons secondaires, donc en définitive les ions.

Ge système a été choisi de préférence à un multiplicateur de particules (sans fenêtre), car il présente sur celui-ci l'avantage de ne pas être contaminé à la longue par les entrées d'air ou des vapeurs résiduelles. De plus, comme les électrons secondaires 
sont émis suivant l'axe vertical de l'enceinte contenant l'appareil, le photomultiplicateur reste fixe lorsque le plateau tourne, ce qui simplifie les connexions électriques.

Lorsque la haute tension n'est pas appliquée à l'électrode à émission secondaire, les ions issus de $\mathrm{F}_{\mathbf{9}}$ ne sont plus déviés et sont recueillis directement dans une cage de Faraday servant, en particulier, à mesurer l'intensité du faisceau incident (de $10^{-9}$ à $10^{-10} \mathrm{~A}$ pour le faisceau direct, mesurés à l'aide d'un picoampèremètre Lemouzy).

2. Méthodes de mesure. - 2.1. MÉthode D'ANALYSE. - Pour que le secteur magnétique fonctionne en simple sélecteur de masses, les ions qu'il reçoit doivent avoir une énergie fixe $E_{\mathrm{a}}$. Il suffit pour cela d'alimenter l'analyseur électrostatique avec une tension également fixe $V_{\mathrm{a}}=k E_{\mathrm{a}}$.

Considérons les ions de masse $M$ sortant de la chambre à une énergie quelconque $E$; si on leur communique une énergie supplémentaire $E_{1}=e V_{1}$, telle que $e V_{1}=E_{\mathrm{a}}-E$, ils traverseront l'analyseur électrostatique, puis le secteur magnétique, si celui-ci est convenablement réglé pour la masse $M$ et l'énergie $E_{\mathrm{a}}$.

Ainsi il est possible d'analyser en énergie une catégorie donnée d'ions diffusés, par variation plus ou moins rapide de $V_{1}, V_{\mathrm{a}}$ restant fixe, ainsi que l'excitation du secteur magnétique.

2.2. Teghniques DE gomptage Des ions analysés. - 2.2.1. Détermination du seuil de discrimination. - Les impulsions délivrées par le P.M. correspondent aux électrons secondaires, ou bien constituent le bruit de fond. Un ion $\mathrm{He}^{+}$incident sur l'électrode haute tension peut donner 1,2 ou 3 électrons secondaires (2,3 en moyenne dans le cas de l'aluminium). Si, par exemple, deux électrons secondaires sont émis simultanément et accélérés à $25 \mathrm{keV}$, les impulsions correspondantes s'ajoutent pour donner une impulsion unique, qui serait celle produite par une seule particule, d'énergie double (50 keV).

Finalement, la distribution des impulsions en amplitude est une courbe présentant un maximum correspondant à une énergie d'environ $50 \mathrm{keV}$. Par contre, la distribution du nombre des impulsions du bruit de fond en fonction de leur amplitude est une courbe rapidement décroissante. L'ensemble du spectre d'amplitude est donné par la figure 7 . Il en résulte que l'on peut, en choisissant une amplitude de discrimination convenable $E_{\mathrm{s}}$, éliminer pratiquement le bruit du P.M., tout en conservant la quasi-totalité des impulsions provenant des ions.

Cependant, les ions analysés ne sont pas les seuls à parvenir au détecteur : quelques ions parasites issus du faisceau incident arrivent jusqu'à la cible à émission secondaire, directement ou par réflexion sur les parois, malgré de nombreux blindages en chicanes. Ils provoquent des impulsions de même amplitude que celles

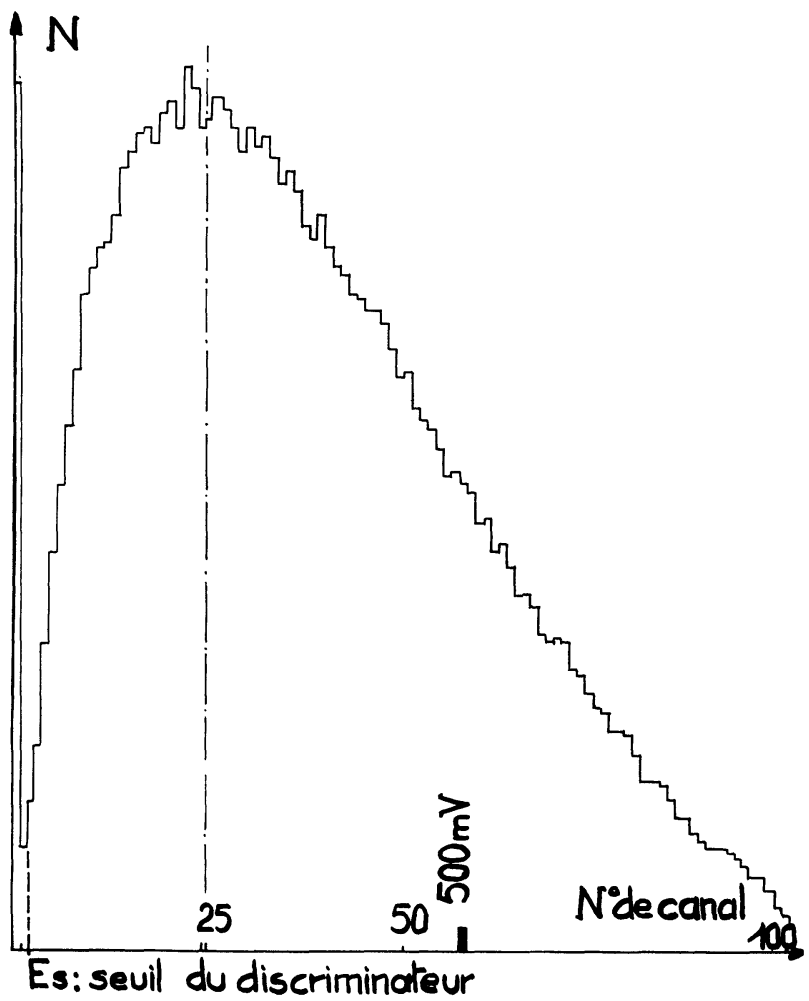

FIG. 7. - Spectre des électrons secondaires issus du détecteur.

fournies par les ions analysés et ne peuvent donc être discriminés. Leur flux est environ de 20 à 30 coups/s. Une protection plus efficace contre ces ions parasites est actuellement à l'étude de façon à ramener le bruit de fond parasite aux environs de $1 \mathrm{coup} / \mathrm{s}$.

2.2.2. Échelle de comptage. — Les impulsions délivrées par le P.M. peuvent être envoyées à un ensemble de comptage comprenant un amplificateur linéaire, un discriminateur d'amplitude, une échelle de comptage et un intégrateur. Le taux maximum de comptage est de 50000 particules/s, correspondant à un courant ionique de quelque $10^{-15} \mathrm{~A}$.

Pour les angles de diffusion supérieurs à 6 ou 7o, le courant ionique est si faible qu'on est essentiellement limité par le bruit de fond provenant des ions parasites; en effet, le signal utile est alors au plus égal à $30 \mathrm{c} / \mathrm{s}$, donc égal ou même inférieur au niveau de bruit. L'obtention des spectres nécessite alors des dispositifs plus élaborés, capables d'extraire le signal $\mathrm{du}$ bruit de fond.

2.2.3. Système « multi-échelles ». - Cet appareil présente 1024 canaux ouverts successivement aux impulsions provenant du détecteur, pendant un temps prédéterminé, par un signal récurrent en dent de scie. Ce même signal, amplifié, est utilisé directement comme tension d'analyse $V_{1}$. Ainsi, à tout instant, on établit une correspondance entre le numéro du canal 
et l'énergie des ions analysés. Les balayages peuvent être répétés autant de fois que l'on désire. Le spectre d'énergie des ions diffusés, accumulé en mémoire, peut être alors visualisé ou enregistré. Cet appareil

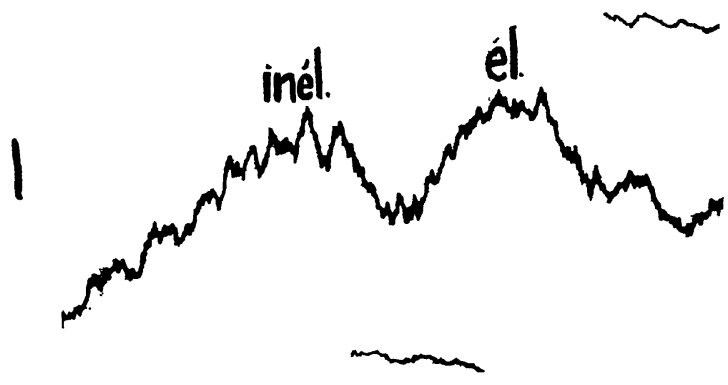

Fig. 8. - Balayage classique : $\theta=5,6^{\circ}$.

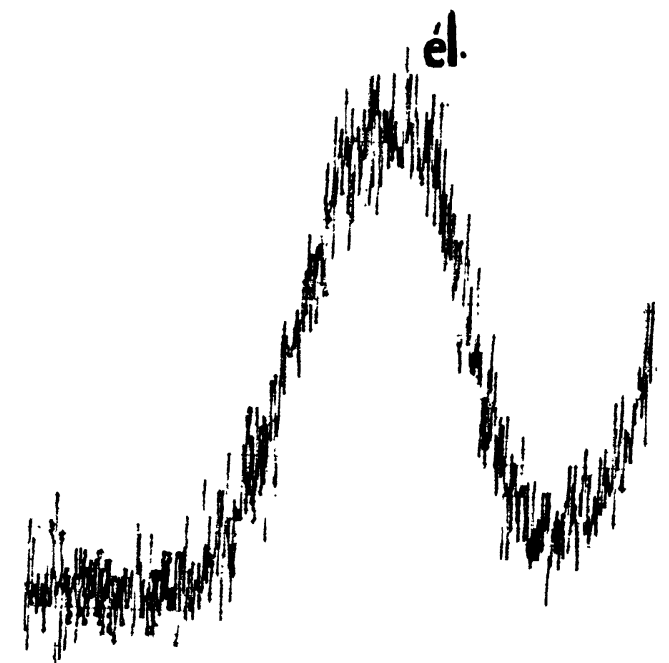

FIG. 9. - Multi-échelles : $\theta=5,6^{\circ}$.
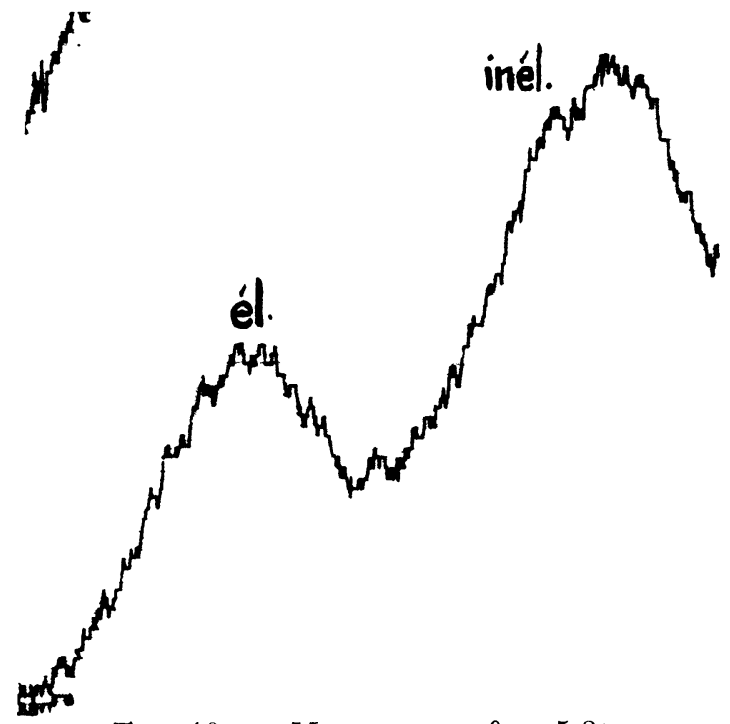

FI' . 10. - Moyenneur : $\theta=5,8^{\circ}$

FIG. 8, 9 et 10. - Spectres des ions diffusés : amélioration du rapport signal sur bruit. permet d'obtenir les spectres dans un intervalle angulaire très étendu.

Aux très faibles angles, il assure de plus la liaison avec la mesure directe du courant par picoampèremètre $\left(I>10^{-12} \mathrm{~A}\right)$.

2.2.4. Moyenneur. - Aux angles supérieurs à $6^{\circ}$, l'amplitude du signal devient égale ou inférieure à celle du bruit de fond. Les impulsions sont alors amplifiées puis intégrées, et le signal continu (noyé dans le bruit) qui en résulte est envoyé dans le moyenneur pendant une série de $n$ balayages. Comme le bruit croît en $\sqrt{n}$ et le signal en $n$, le spectre apparaît peu à peu et se dessine d'autant mieux que $n$ est grand. Grâce à la stabilité du faisceau, la durée totale de balayage a pu être portée à 1 heure (32 balayages de $2 \mathrm{mn}$ ).

Les figures 8, 9 et 10 donnent les spectres obtenus respectivement par les moyens classiques (intégrateur), à l'aide du multi-échelles et à l'aide du moyenneur.

3. Résultats. - Les premières expériences entreprises ont porté sur la mesure des sections efficaces différentielles relatives de collision élastique d'ions $\mathrm{He}^{+}$ sur des atomes $\mathrm{He}$, dans le domaine d'énergie incidente $0,5 \mathrm{keV}-3 \mathrm{keV}$.

Les ions projectiles ayant une énergie $E_{0}$ donnée, l'ensemble analyseur est réglé sur le faisceau incident, à l'angle $\theta=0$.

La pression $P$ du gaz cible est fixée à une valeur située dans la zone de variation linéaire du courant diffusé, de telle sorte qu'il ne se produise pas de chocs multiples; $P$ est comprise entre $10^{-4}$ et $10^{-3}$ Torr.

On fait alors tourner le système d'analyse d'un angle $\theta$ et on enregistre le spectre d'énergie des particules diffusées (voir, par exemple, la figure 11). On peut observer sur ces spectres :

- Un premier pic (A), qui représente les ions diffusés élastiquement et auxquels correspond la perte d'énergie :

$$
\left.\Delta E_{\mathrm{el}} \simeq E_{0} \cdot \theta^{2} \quad \text { (pour } \theta \text { faible }\right) .
$$

- Un second pic (B) représente une diffusion inélastique. Il comprend une perte correspondant à l'excitation de l'atome cible :

$$
\overline{\mathrm{He}}^{+}+\mathrm{He} \rightarrow \overline{\mathrm{He}}^{+}+\mathrm{He}^{*}
$$

et à l'ionisation de l'atome cible :

$$
\overline{\mathrm{He}}^{+}+\mathrm{He} \rightarrow \overline{\mathrm{He}^{+}}+\mathrm{He}^{+}+\text {e. }
$$

- Un pic $(\mathrm{G})$ représente l'excitation de l'ion incident

$$
\overline{\mathrm{He}^{+}}+\mathrm{He} \rightarrow\left(\mathrm{He}^{+}\right)^{*}+\mathrm{He} .
$$



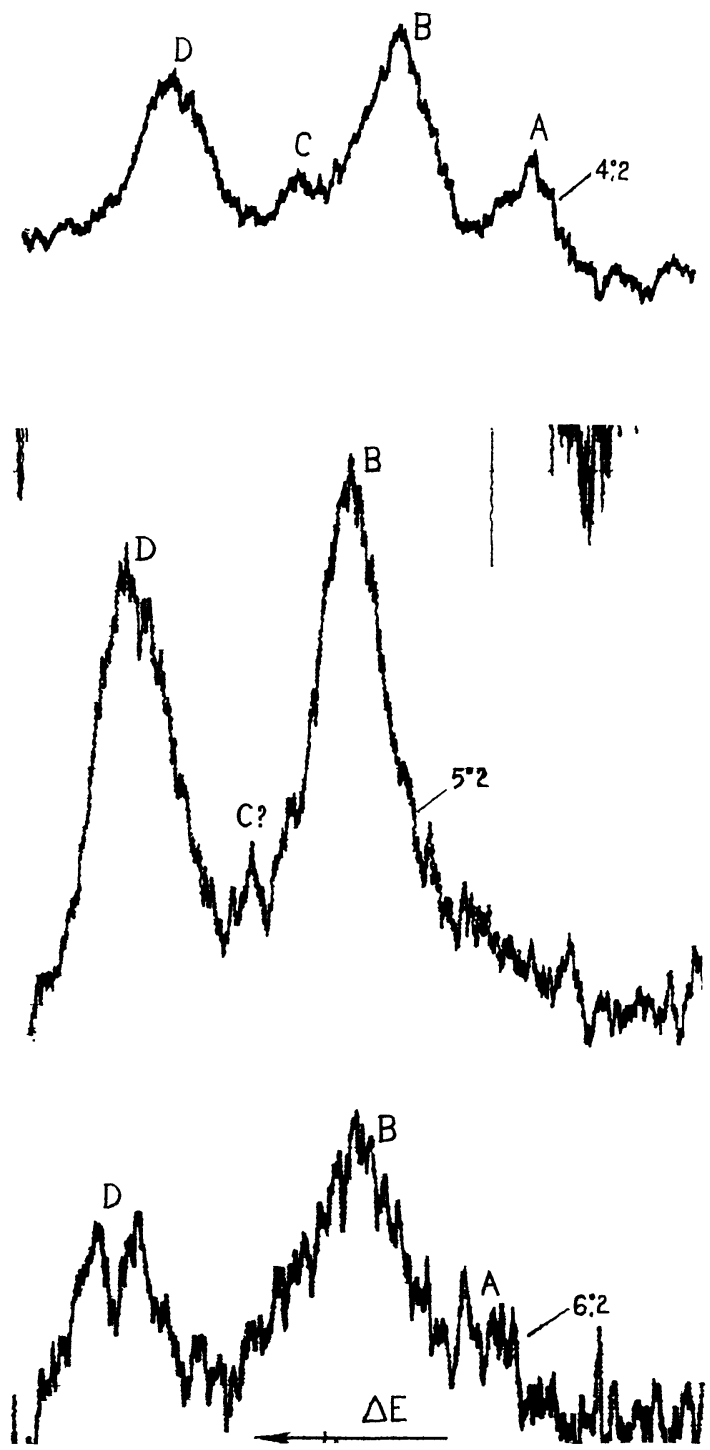

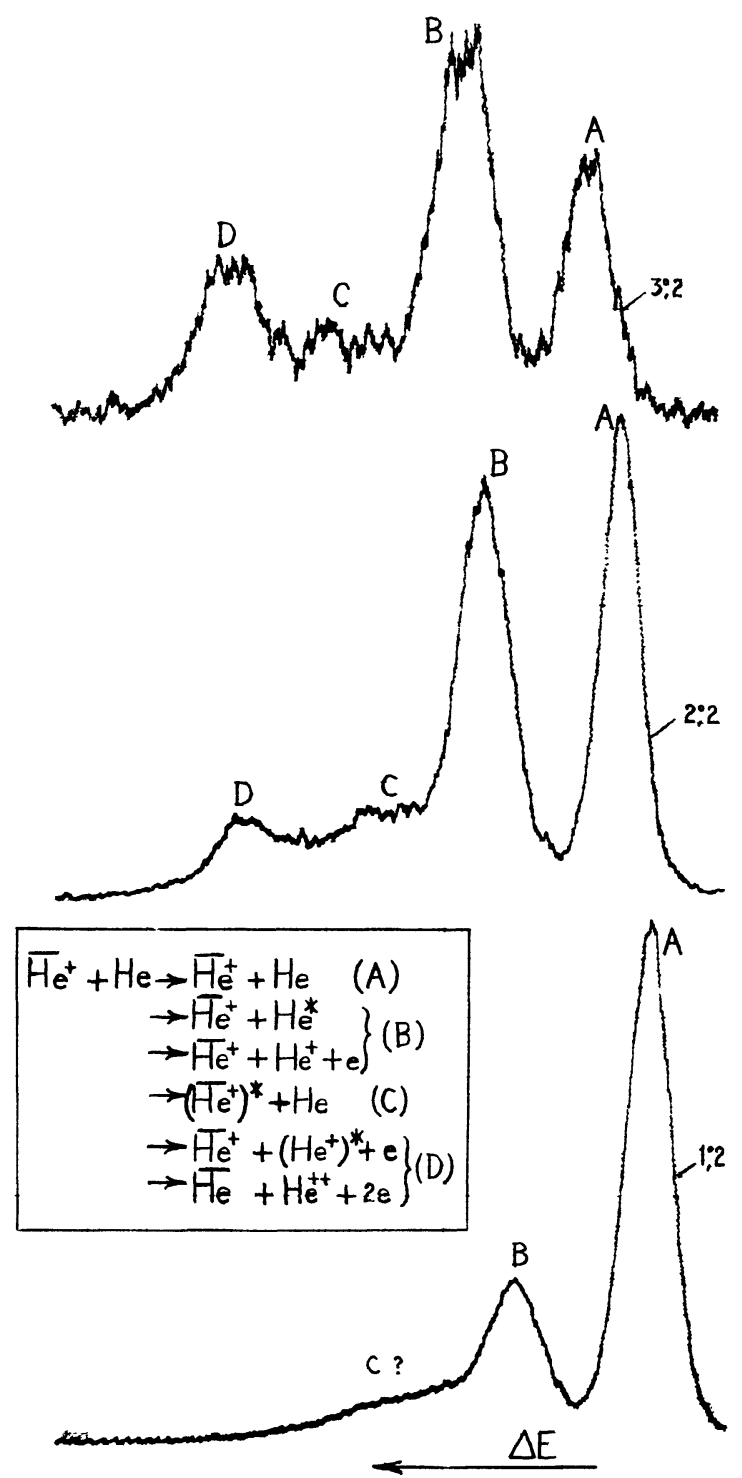

FIG. 11. - Évolution relative des pics de diffusions élastique et inélastique en fonction de l'angle de diffusion.

- Enfin, un pic (D) représente l'ionisation puis l'excitation de l'atome cible :

$$
\overline{\mathrm{He}^{+}}+\mathrm{He} \rightarrow \overline{\mathrm{He}^{+}}+\left(\mathrm{He}^{+}\right)^{*}+\mathrm{e}
$$

et l'ionisation double de l'atome cible :

$$
\overline{\mathrm{He}^{+}}+\mathrm{He} \rightarrow \overline{\mathrm{He}^{+}}+\mathrm{He}^{2+}+2 \mathrm{e}
$$

Pour ces divers processus inélastiques, si $Q$ est la variation de l'énergie interne totale, la perte $\Delta E_{\text {in }}$ observée est :

$$
\Delta E_{\mathrm{in}}=\Delta E_{\mathrm{el}}+Q\left(1+\frac{Q}{4 E_{0} \cos ^{2} \theta}\right)
$$

Nous nous sommes limités, dans un premier temps, à l'étude des sections efficaces élastiques.

En mesurant l'aire du pic élastique, on obtient un nombre proportionnel au nombre d'ions diffusés élastiquement. En multipliant l'aire par $\sin \theta$, on obtient la section efficace différentielle élastique en valeur relative (voir Appendice I).

La section efficace $\Sigma(\theta)$ obtenue présente une série d'oscillations analogues à celles observées par D. C. Lorents et W. Aberth [2]. Leur étude systématique et l'interprétation qu'on peut en donner seront publiées prochainement. 


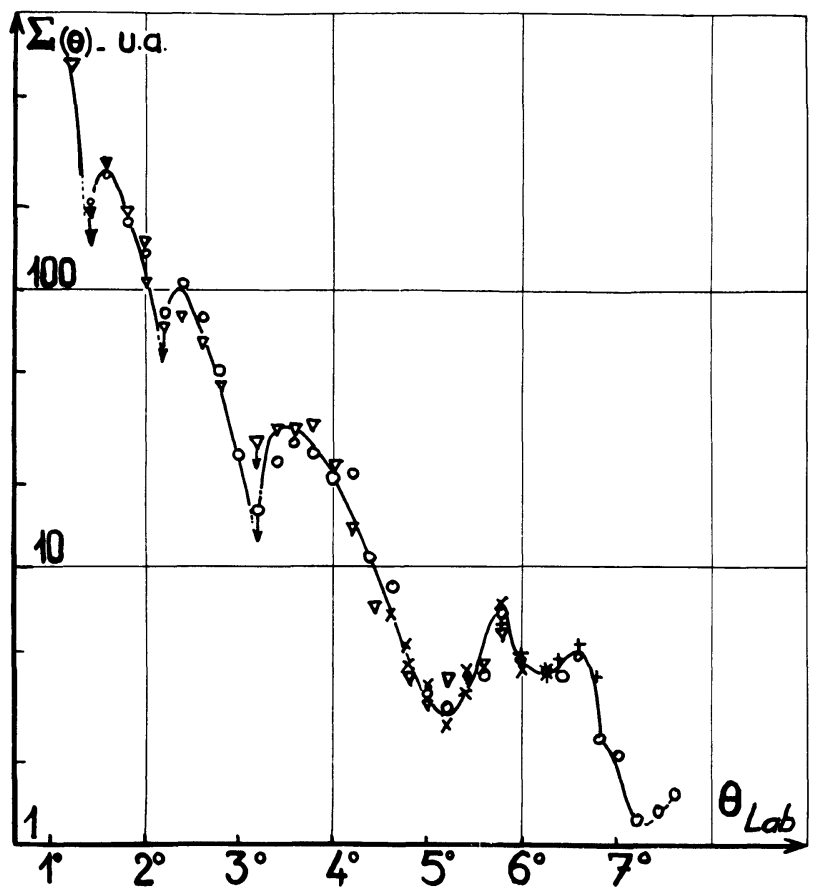

FIG. 12. - Section efficace élastique différentielle $\Sigma(\theta) \mathrm{He}^{+}$ sur $\mathrm{He}$ à $1,5 \mathrm{keV}$.

Remerciements. - Cette étude a été entreprise dans le cadre d'un contrat D.R.M.E. Nous remercions cet organisme pour le soutien financier qu'il nous a accordé.

\section{APPENDICE I}

Calcul du rapport $I_{\mathrm{s}} / I_{0}$. $-\mathrm{Si} I_{0}$ est le courant entrant dans la chambre de collision, $I_{\mathrm{s}}$ le courant sortant, le rapport $I_{\mathrm{s}} / I_{0}$ est donné par :
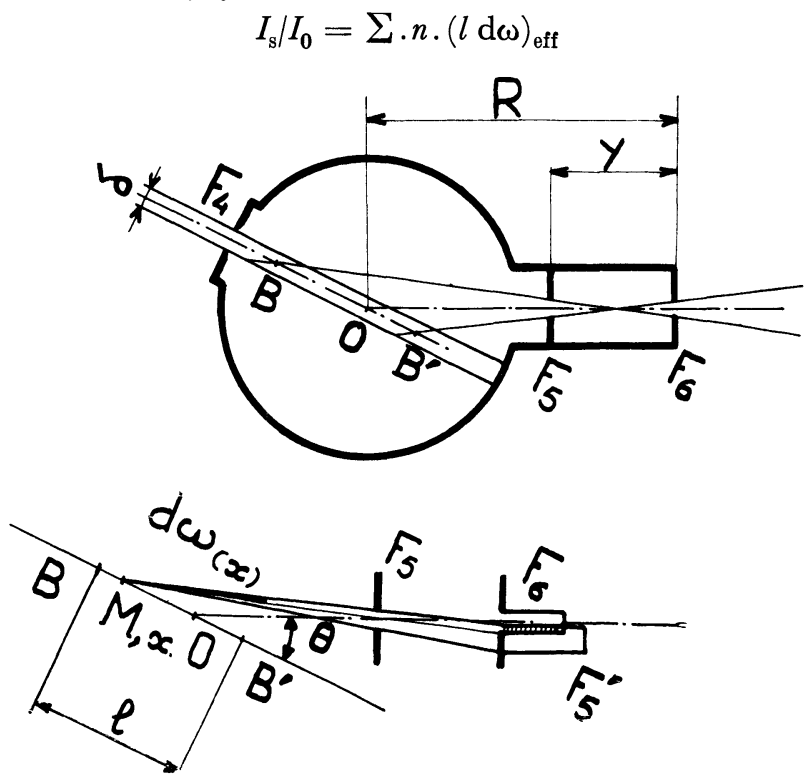

FIG. 13. - Détermination du volume de collisions. où $\Sigma$ est la section efficace différentielle pour le processus considéré et $n$ le nombre de particules cibles par unité de volume :

$$
n=3,885 \times 10^{16} P \text { particules } / \mathrm{cm}^{3}
$$

$P$ étant la pression du gaz cible en torr. Nous prendrons dans ce qui suit : $P=10^{-3}$ torr. La quantité $(l \mathrm{~d} \omega)_{\text {eff }}$ est calculée comme suit [10] (voir fig. 13).

Soit $w$ la largeur des fentes $F_{5}$ et $F_{6}, h$ leur hauteur commune, $y$ la distance $\mathrm{F}_{5} \mathrm{~F}_{6}, R$ la distance du centre $\mathrm{O}$ de la chambre à $\mathrm{F}_{6}, \theta$ l'angle de diffusion.

Les fentes $F_{5} F_{6}$ déterminent le volume utile de collision $\left(\mathrm{BOB}^{\prime}\right)$ qui contribue seul au courant de particules diffusé au travers de $F_{5}$ et $F_{6}$. Aux extrémités BB' du volume utile, l'angle solide dans lequel sont émises les particules qui atteindront l'analyseur est nul; il est, par contre, maximum pour un élément de volume placé au voisinage de $\mathrm{O}$.

On pose $l=\mathrm{BB}^{\prime}$, et on suppose la largeur $\delta \mathrm{de}$ la fente d'entrée $\mathrm{F}_{4}$ très inférieure à $l$. Pour un élément de volume d'épaisseur $\mathrm{d} x$ repéré par l'abscisse $x$, l'angle solide utile est $\mathrm{d} \omega(x)$, de sommet $M$ et s'appuyant sur le contour de la surface $S(x)$ commune aux deux rectangles $\left(F_{6}\right)$, seconde fente, et $\left(F_{5}^{\prime}\right)$ homothétique de $\left(F_{5}\right)$ dans l'homothétie de centre $M$ faisant passer du plan de $\left(\mathrm{F}_{5}\right)$ au plan de $\left(\mathrm{F}_{6}\right)$.

En supposant $x \ll R$, on peut écrire :

$$
\mathrm{d} \omega(x) \simeq \frac{S(x)}{R^{2}}
$$

et, par raison d'homothétie : $l \cdot \sin \theta=w \frac{2 R-y}{y}$.

L'angle solide moyen est donc :

$$
\mathrm{d} \omega_{\mathrm{m}}=\frac{1}{l} \cdot \frac{1}{R^{2}} \int_{-l / 2}^{+l / 2} S(x) \cdot \mathrm{d} x .
$$

Après intégration, on trouve :

$$
(l \mathrm{~d} \omega)_{\mathrm{eff}}=l \cdot \mathrm{d} \omega_{\mathrm{m}}=\frac{w^{2} h}{R \cdot y \cdot \sin \theta} .
$$

En reportant cette expression dans $I_{\mathrm{s}} / I_{0}$, on obtient :

$$
I_{\mathrm{s}} / I_{0}=1,45 \times 10^{8} \frac{\sum\left(\mathrm{cm}^{2}\right)}{\sin \theta} .
$$

On peut, pour avoir un ordre de grandeur de $I_{\mathrm{s}} / I_{0}$, utiliser les résultats obtenus par Lane et Everhart [11] sur la section efficace différentielle de collision élastique d'ions $\mathrm{Ar}^{+}$de $2 \mathrm{keV}$ sur des atomes Ar. On obtient alors les résultats suivants :

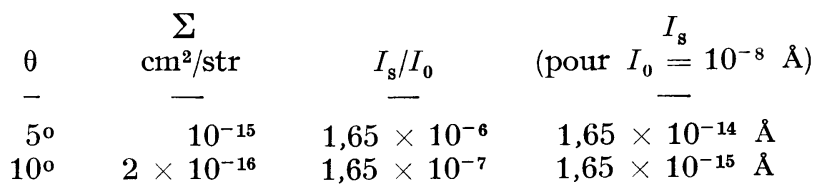

Ces valeurs de $I_{\mathrm{s}}$, déjà très faibles, sont encore supérieures aux valeurs réelles, puisqu'elles font abstraction de l'analyse en énergie. Si les particules qui atteignent le détecteur final ont une énergie 
comprise entre $E$ et $E+\Delta E$, le courant final $I_{\mathrm{f}}$ sera tel que :

$$
I_{\mathrm{f}} / I_{0}=\Sigma^{\prime} \cdot n \cdot(l \mathrm{~d} \omega)_{\mathrm{eff}} \cdot \Delta E
$$

où $\Sigma^{\prime}$ est la section efficace " doublement différentielle » $: \Sigma^{\prime}=\frac{\partial^{2} \sigma}{\partial \omega . \partial E}$.

Le problème est en fait encore plus compliqué, pour la raison que, dans un processus déterminé - élastique par exemple - l'énergie $E$ des particules est liée à la direction dans laquelle elles sont diffusées. Ainsi, à la dispersion en direction $\mathrm{d} \omega($ ou $\mathrm{d} \theta$ ) correspond une dispersion en énergie $\delta E$, et les résultats sont différents selon que $\delta E$ est supérieur ou inférieur à l'acceptance en énergie $\Delta E$ de l'analyseur électrostatique. On peut montrer que :

$$
I_{\mathrm{f}}=\left\{\begin{array}{lll}
I_{\mathrm{s}} & \text { si } & \Delta E>\delta E \\
\frac{\Delta E}{\delta E} . I_{\mathrm{s}} & \text { si } & \Delta E<\delta E .
\end{array}\right.
$$

L'acceptance en énergie $\Delta E$ est donnée par la relation $\Delta E \simeq E \cdot \frac{w_{8}}{R_{\mathrm{a}}}, w_{8}$ étant la largeur de $\mathrm{F}_{8}$ et $R_{\mathrm{a}}$ le rayon de la trajectoire dans l'analyseur électrostatique.

\section{APPENDICE II}

Anomalies au voisinage des minimums de $\Sigma$. Aux minimums de la section efficace, on remarque que les pics de diffusion élastique présentent une structure double. Prenons par exemple (fig. 14) les spectres obtenus à $\theta=1,8^{\circ}, 2^{\circ}, 2,2^{\circ}$ au voisinage du minimum que présente $\Sigma$ à $\theta=2^{\circ}$ (pour $E_{0}=2,5 \mathrm{keV}$ ).

Le dédoublement du pic élastique est la conséquence de la résolution angulaire limitée $\delta \theta$ de l'appareil. En effet, lorsque l'analyseur est placé dans une position angulaire $\theta$ correspondant rigoureusement à un minimum de $\Sigma$, le pic élastique devrait avoir une amplitude très faible. En fait, on détecte, en plus des particules diffusées en $\theta=2^{\circ}$, toutes celles diffusées d'une part entre $2^{\circ}-\frac{\delta \theta}{2}$ et $2^{\circ}$, d'autre part entre $2^{\circ}$ et $2^{\circ}+\frac{\delta \theta}{2}$, angles pour lesquels la section efficace est plus grande. La perte d'énergie $\Delta E_{\mathrm{el}}$ étant une fonc-
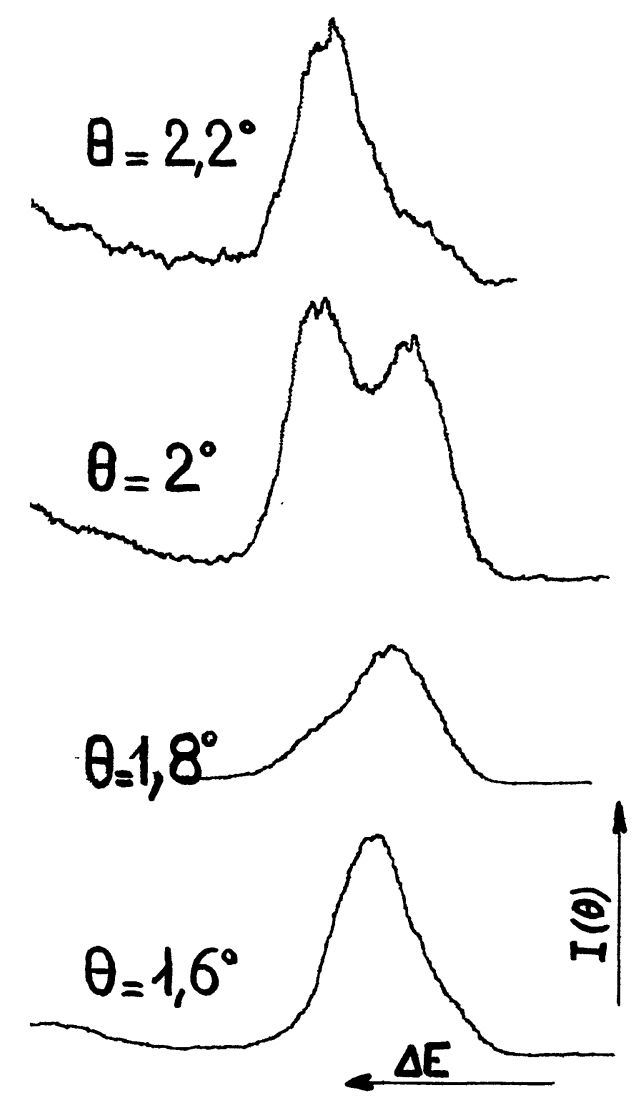

Fig. 14. - Dédoublement du pic au voisinage d'un minimum de $\Sigma(\theta)$.

tion croissante de $\theta$, quand on effectue l'analyse en énergie, le nombre d'ions ayant subi la perte d'énergie correspondant à $\theta_{\mathrm{m}}=2^{\circ}$ est beaucoup plus faible que celui des ions ayant subi une perte légèrement supérieure $\left(\right.$ pour $\left.\theta_{\mathrm{m}}+\frac{\delta \theta}{2}\right)$ ou légèrement inférieure $\left(\theta_{\mathrm{m}}-\frac{\delta \theta}{2}\right)$, ce qui explique la structure double du pic élastique. On peut ainsi, par observation des pics doubles, localiser avec précision les minimums de $\Sigma(\theta)$.

Manuscrit reçu le 14 juin 1967.

\section{BIBLIOGRAPHIE}

[1] Consulter, par exemple, la bibliographie donnée par : McDANIEL (E. W.), Collisions phenomena in ionized gases, J. Wiley, New York, 1964.

[2] LORENTS (D. C.), ABERTH (W.), Phys. Rev., 1965, 139, 1017.

[3] MCClure (J. W.), Phys. Rev., 1965, 140, 769.

[4] Carlson (C. E.), Magnuson (G. D.), Rev. Sci. Instr., 1962, 33, 905.

[5] Judd (D. L.), Rev. Sci. Instr., 1950, 21, 213.

[6] SNyder (C. W.), RUbiN (S.), FOWLER (W. A.), LAURITSEN (C. C.), Rev. Sci. Instr., 1950, 21, 852.
[7] Hughes (A. L.), Rojansky (V.), Phys. Rev., 1929, 34, 284.

[8] DALy (N. R.), Rev. Sci. Instr., 1960, 31, 264. Afrosimov (V. V.) et al., Sov. Phys. Tech. Phys. (traduction anglaise), 1961, 5, 1378.

[9] ARDUinI (M.), D.E.S., Orsay, 1966.

[10] Jordan (E. B.), Brod (R. B.), Phys. Rev., 1933, 43, 112.

[11] Lane (J. H.), Everharit (E.), Phys. Rev., 1960, 120, 2064. 